Summer Meeting, 6-9 July 2015, The future of animal products in the human diet: health and environmental concerns

\title{
With age spontaneous baroreflex sensitivity diminishes after acute fat ingestion
}

\author{
C.I. Wright ${ }^{1}$, H. Ruediger ${ }^{2}$ and R. Draijer ${ }^{3}$ \\ ${ }^{1}$ Red Pharm, Cold Meece Estate, Staffordshire, ST15 OSP, ${ }^{2}$ Institute of Occupational and Social Medicine, \\ University of Technology, Dresden, Germany and ${ }^{3}$ Nutrition and Health, Unilever R\&D, Vlaardingen, The Netherlands
}

Baroreflex sensitivity (BRS) is a marker of circulatory autonomic control ${ }^{(1)}$ and shown to be attenuated in smokers ${ }^{(2,3)}$. Previous studies found that acute fat ingestion had no effect on $\mathrm{BRS}^{(4)}$. However, the effect of fat ingestion on spontaneous BRS as age increases remains unclear and this was investigated in the current study.

30 male smokers aged 19 to 45 years of age were recruited for a randomised, placebo-controlled crossover designed study. A tonometer was used to average three 30 -second radial pulse wave form tracings, four hours after subjects ingesting $50 \mathrm{ml}$ of whipping cream containing $50 \mathrm{~g}$ of fat (high fat) or $50 \mathrm{ml}$ of milk containing $5 \mathrm{~g}$ of fat (low fat). Variations in beat-to-beat values of systolic blood pressure (SBP) and pulse interval (PI) were analysed to acquire an estimate of spontaneous BRS and cardiac parasympathetic activity.

Group analysis showed that cream increased SBP by $2 \mathrm{mmHg}$ and decreased PI by $29 \mathrm{~ms}(\mathrm{P}<0 \cdot 05)$, but BRS and cardiac parasympathetic activity remained unchanged. A further analysis was conducted by plotting age against BRS to assess the effect of fat. In the low fat group, no linear correlation was found between age and BRS $(\mathrm{r}=+0 \cdot 08, \mathrm{P}=0 \cdot 70$; Pearson correlation coefficient) and cardiac parasympathetic activity $(\mathrm{r}=+0 \cdot 38, \mathrm{P}=0.08)$. In contrast, in the high fat group, a negative linear correlation existed between BRS and age $(\mathrm{r}=-0.45, \mathrm{P}=0.02)$, but not between cardiac parasympathetic activity and age $(\mathrm{r}=+0 \cdot 25, \mathrm{P}=0 \cdot 22)$.

In male smokers per se, the acute ingestion of fat has minor effects on the cardiovascular system. Moreover, sub-analysis may imply that with age, the ingestion of high fat may markedly reduce BRS and impair short-term autonomic control of blood pressure.

1. Studinger P, Mersich B, Lenard Z et al. (2004). Effect of vitamin E on carotid artery elasticity and baroreflex gain in young, healthy adults. Auton Neurosci 113, 63-70.

2. Arosio E, De Marchi S, Rigoni A et al. (2006). Effects of smoking on cardiopulmonary baroreceptor activation and peripheral vascular resistance. Eur J Clin Invest 36, 320-325.

3. Kardos A, Watterich G, de Menezes R et al. (2001). Determinants of spontaneous baroreflex sensitivity in a healthy working population. Hypertension 37, 911-916.

4. Wright CI, Ruediger H, Kroner CI et al. (2009). Acute autonomic effects of vitamins and fats in male smokers. Eur J Clin Nutr 63 (2), $246-252$. 